\title{
MEXICO CITY'S AIRBNB LISTING PRICE ANALYSIS USING REGRESSION
}

\author{
Daniela A. Gomez-Cravioto, Ramon E. Diaz-Ramos, Virginia I. Contreras-Miranda \\ and Francisco J. Cantu-Ortiz \\ School of Engineering and Science, Tecnológico de Monterrey, Monterrey, México
}

\begin{abstract}
The AirBnb platform provides users with the option of renting their vacant spaces as tourist accommodations and competing with traditional accommodation enterprises. However, since AirBnb gives the user the freedom to establish the price of their listing, a challenge is placed on the owner to determine the most appropriate number. This study analyses the information from listings in Mexico City to determine how the listing attributes can be used as predictors for a new listing's price. The study uses statistical methods and machine learning techniques to analyze the information scraped from AirBnb's website, which is publicly available at the Inside AirBnb webpage. In this work, an experiment was made to compare a quantile regression, logistic regression, and a generalized additive model to find the most suitable technique for predicting an AirBnb listing's price. The models were compared based on the residual standard error, $\mathrm{R}$ squared, and AIC. The results show that the generalized additive model provides the best fit for the dataset explaining $60 \%$ of the variance.
\end{abstract}

\section{KEYWORDS}

AirBnb, Mexico, CRISP-DM, Logistic Regression, GAM, Quantile Regression

\section{INTRODUCTION}

The sharing economy has created a great number of opportunities for Smart Cities and communities around the world to create a better and smarter working and living environment (Tedjasaputra and Sari, 2016). The smart city concept covers a variety of industries, one of these is the tourism industry. Bringing smartness into tourism destinations requires the destinations to facilitate information exchange amongst stakeholders to enhance the decision-making process. This is helping the tourism service providers provide new improved services to tourists (Jasrotia and Gangotia, 2018). An example of this is the AirBnb business.

Since its creation in 2007, the company has experienced explosive growth, from being an online bed and breakfast company to a leading peer-to-peer hospitality corporation. The company now offers its services in over eighty thousand cities worldwide and it is continuously growing. This expansion is considered a part of a large rise in the sharing economy which has represented an inordinate source of strategic disruption and business successes over the last decade (Aaker, 2019). The service offers people with a free-living space a new source of income and for guests an affordable option.

One challenge that hosts in AirBnb experience is determining their optimal nightly price for their listings. This is a problem that can be approached with the use of machine learning techniques. There is existing literature focused on price predictions. We analyze six works that are the most pertinent to this study. First, Mason and Quigley (1996) analyzed the decline in condominium house values in downtown Los Angeles with the use of a generalized additive model (GAM). Secondly, Zietz et al. (2008) determined the relationship of a particular housing characteristic with selling price with quantile regression. Raymond et al. (2016) defined three types of neighborhood housing markets in the Atlanta region based on the zip code level, their levels of volatility, and stability before, during, and after the housing crisis with Multinomial Logistic Regression. Yu and Wu (2016) also used Multinomial Logistic Regression to predict house prices given explanatory variables that cover many aspects of residential houses. Finally, a study performed by Perez-Sanchez et al. (2018) identified the determinants of AirBnb accommodation prices by estimating a hedonic price model that adopted quantile regression methods. 
We consider this study adds to the current literature by focusing on regression techniques and comparing these in a dataset from Mexico City since we did not find previous related work performed in this country. The main objective of this paper is to build and evaluate several regression models to suggest the most convenient pricing for a new AirBnb host's listing taking into account the listing features. The goal is to allow hosts to understand which features are the most important for price assignment and understand how the price changes based on seasonality and holidays in Mexico. Specifically, the research questions that we are trying to solve are the following: (1) What are the most common AirBnb property types in Mexico City available for renting?; (2) What is the average price of the properties?; (3) What is the most expensive location in Mexico City?; (4) What time of the year are AirBnb's most popular in Mexico City? Is there any holiday that spikes pricing?; (5) What are the most important features of a listing in Mexico city, and how does this affect the price?; (6) Can the selected features be used to create a data-based model that can predict the price of a new listing? The machine learning techniques used in this paper are the following: quantile regression, logistic regression, and generalized additive model.

\section{RELATED WORK}

In this section, we present related studies focused on house pricing; these are shown in Table 1. Firstly, Perez-Sanchez et al. (2018) identified the determinants of AirBnb accommodation prices by evaluating housing characteristics (advertisement, features, and location) which adopted quantile regression methods. Secondly, Zietz et al. (2008) determined the relationship of particular housing characteristics (physical, geographic, and neighborhood characteristics) with selling price with Quantile Regression. Mason and Quigley (1996) analyzed the decline in condominium house values in downtown Los Angeles with the use of a GAM model. The dataset contained a sample of 843 condominium sales recorded for 12 years. Finally, Raymond et al. (2016) defined three types of neighborhood housing markets in the Atlanta region based on the zip code level, their levels of volatility and stability before, during, and after the housing crisis with Multinomial Logistic Regression.

Table 1. Evaluation of the related works models

\begin{tabular}{|c|c|c|c|}
\hline Source & Variables & Model & Results \\
\hline $\begin{array}{l}\text { Affuso et al. } \\
\text { (2019) }\end{array}$ & $\begin{array}{l}\text { Noise levels, property, and neighborhood } \\
\text { characteristics. }\end{array}$ & GAM & $\mathrm{AIC}=14,066$ \\
\hline $\begin{array}{l}\text { Perez-Sanchez } \\
\text { et al. (2018) }\end{array}$ & $\begin{array}{l}\text { Price and characteristics of the } \\
\text { accommodation, advertisement, host } \\
\text { features, environmental and location } \\
\text { characteristics. }\end{array}$ & $\begin{array}{l}\text { Quantile } \\
\text { Regression }\end{array}$ & $\begin{array}{l}\text { Pseudo R2 } \\
\text { Q.10=.479, Q.25=.415, Q.50=.378 } \\
\text { Q.75=.378, Q.90 = .397 }\end{array}$ \\
\hline $\begin{array}{l}\mathrm{Yu} \text { and } \mathrm{Wu} \\
(2016)\end{array}$ & $\begin{array}{l}79 \text { features such as areas of the houses, } \\
\text { types of floors, and the number of } \\
\text { bathrooms. }\end{array}$ & $\begin{array}{l}\text { Multinomial } \\
\text { Logistic } \\
\text { Regression }\end{array}$ & $\mathrm{RMSE}=.5587$ \\
\hline $\begin{array}{l}\text { Zietz et al. } \\
(2008)\end{array}$ & $\begin{array}{l}33 \text { attributes that include physical, } \\
\text { geographic, and neighborhood } \\
\text { characteristics of each house. }\end{array}$ & $\begin{array}{l}\text { Quantile } \\
\text { Regression }\end{array}$ & $\begin{array}{l}\text { Pseudo R2 } \\
\text { Q. } 10=.523, \text { Q. } 20=.531, \mathrm{Q} .30=.543 \\
\text { Q.40=.548, Q.50=.554, Q.60=.565 } \\
\text { Q.70=.568, Q. } 80=.569, \mathrm{Q} .90=.549\end{array}$ \\
\hline $\begin{array}{l}\text { Mason and } \\
\text { Quigley (1996) }\end{array}$ & Condominium sales. & GAM & $R^{2}=.759$ \\
\hline $\begin{array}{l}\text { Raymond et al. } \\
\text { (2016) }\end{array}$ & $\begin{array}{l}\text { Housing market and demographic } \\
\text { variables of } 137 \text { zip codes. }\end{array}$ & $\begin{array}{l}\text { Multinomial } \\
\text { Logistic } \\
\text { Regression }\end{array}$ & Pseudo R2 $=.679$ \\
\hline
\end{tabular}

\section{METHODS AND METHODOLOGY}

This study uses the CRISP-DM methodology as a standard approach to perform data mining analysis. CRISP-DM stands for "Cross-Industry Standard Process for Data Mining” (Chapman et al., 2000). It consists of a six-step process, including Business Understanding, Data Understanding, Data Preparation, Modelling, 
Evaluation, and Deployment. Each phase consists of second-level tasks which aim to cover all the data mining applications. The initial intention of this methodology was to develop modeling techniques for industry. In this study, we will illustrate the CRISP-DM process and apply it to the problem of predicting new listings in the Mexico AirBnb dataset.

\subsection{Quantile Regression}

The advantage of using the quantile regression technique is that it aids in understanding the relationship between variables outside of the mean of the data, making it useful for understanding outcomes that are non-normally distributed and which do not have linear relationships with the predictor variables (Koenker 2012).

Quantile regression methods offer a mechanism for estimating models for the conditional median function, as well as other conditional quantile functions. It is highly used in econometrics studies, for wage determinants, discrimination effects, as well as trends in income inequality. Due to the nature of the outcome variable, there have been previous studies that have used quantile regression to predict the price of AirBnb listings for other cities (Perez-Sanchez et al., 2018) (Wang and Nicolau, 2017). This study uses quantile regression to predict the price of a new AirBnb listing in Mexico City because it provides a more comprehensive description of the conditional distribution and measures the effects of individual explanatory variables on the whole distribution (Wang and Nicolau, 2017).

\subsection{Logistic Regression}

The method of logistic regression can be used both as a classification technique and a regression technique, it depends on the scenario in which it is used (Dutt et al., 2019). For logistic regression, the dependent variable can be binary $(1,0)$ or multinomial (more than 2 labels) and the independent variables can be either nominal or numerical. This machine learning technique uses the sigmoid function (Figure 1) and obtains the values of the coefficients ( $\beta$ ) by using the maximum likelihood. The function outputs a value between 0 and 1 , which corresponds to the probability of a Bernoulli random variable X (Deisenroth et al., 2020).

$$
p(X)=\frac{e^{\beta_{0}+\beta_{1} X}}{1+e^{\beta_{0}+\beta_{1} X}}
$$

\subsection{Generalized Additive Model}

The generalized additive model (GAM) provides the advantages of allowing a non-linear relation function of independent variables. It can be used with both numeric and nominal dependent variables, however only the numeric can be replaced with a nonlinear function (smooth) which gives it more flexibility to fit the data. The limitation of GAMs is that the model is restricted to be additive, so some variable interactions can be missed. The model can be represented as Function 2, where the $\mathrm{f}_{\mathrm{j}}\left(\mathrm{x}_{\mathrm{ij}}\right)$ represents the nonlinear function or smoothing term (James et al., 2013).

$$
y_{i}=\beta_{0}+\sum_{j=1}^{p} f_{j}\left(x_{i j}\right)+\epsilon_{i}
$$

\section{DATA UNDERSTANDING AND PREPARATION}

The goal of this research is to make a predicting model to suggest the most convenient pricing for AirBnb host's listing with respect to the attributes of accommodation obtained from the Mexico City dataset. More specifically, the research questions that we are trying to solve in this study are the following:(1) What are the most common AirBnb property types in Mexico City available for renting?; (2) What is the average price of the properties?; (3) What is the most expensive location in Mexico City?; (4) What time of the year are AirBnb's listings most popular in Mexico City? Is there any holiday that spikes pricing?; (5) What are the most important features of a listing in Mexico city, and how does this affect the price?; (6) Can the selected features be used to create a data-based model that can predict the price of a new listing? 


\subsection{The Dataset}

This study analyzes the information from the Mexico City AirBnb dataset. The data was scraped from AirBnb's website and publicly available from the Inside AirBnb website (Cox, 2017). Two datasets were collected: "listing.csv", which contains demographic information from the AirBnb listings and the "calendar.csv", that contains the dates and prices of each listing. The listing data is a snapshot of searchable listings from November 25 of 2019. The Mexico City original listing file contains 20,571 AirBnb listings and 106 features.

It was identified that some of the attributes did not contain relevant information for our prediction model, in those cases the feature was eliminated. The 29 features that were selected for the final dataset are shown in Table 2.

Table 2. Features

\begin{tabular}{|c|c|}
\hline Feature & Feature Type \\
\hline host_since, last_review & date \\
\hline $\begin{array}{l}\text { host_response_time, neighbourhood_cleansed, property_type, room_type, bed_type, } \\
\text { cancellation_policy }\end{array}$ & factorial \\
\hline $\begin{array}{l}\text { host_response_rate, latitude, longitude, accommodates, bathrooms, bedrooms ,beds, } \\
\text { price, guests_included, extra_people, minimum_nights, maximum_nights, } \\
\text { availability_365, number_of_reviews, review_scores_rating, calculated_host_list, } \\
\text { review_per_month }\end{array}$ & numerical \\
\hline host_is_superhost, host_has_profile_pic, host_identity_verified, instant_bookable & logical \\
\hline
\end{tabular}

\subsection{Data Cleaning}

There were missing values present in some of the attributes, those attributes with more than $50 \%$ of their total data being missing values were removed from the data set, and the rest were replaced by using an imputation method. Imputation works by calculating the mean/median/mode of the non-missing values of an attribute and then replacing the missing values with this value. The mean was used for numeric data, the median for the numeric data with extreme values, and the mode for nominal data. The attributes "host response time", "host response rate", "reviews score rating", and "reviews per month" had $16 \%, 16 \%, 21 \%$, and $21 \%$ of missing values respectively. The mean was used to fill out missing values for the variable "review per month"; the median, which is a more robust statistic for variables with extreme values, was used to replace the missing values of "host response rate" and "review score rating"; and for the categorical attribute, "host response time", the mode was used.

It was observed, through a boxplot (Figure 1.a) that the target variable "Price" contained outliers. These values can influence negatively in our model. For this reason, we performed the method of winsorization. This method involves the replacement of the outlier values with the most extreme retained values (Wilcox, 2005). In this study, we used the quantiles 3 and 97 to define the limits of the extreme values and those outside these limits were replaced (Figure 1.b).

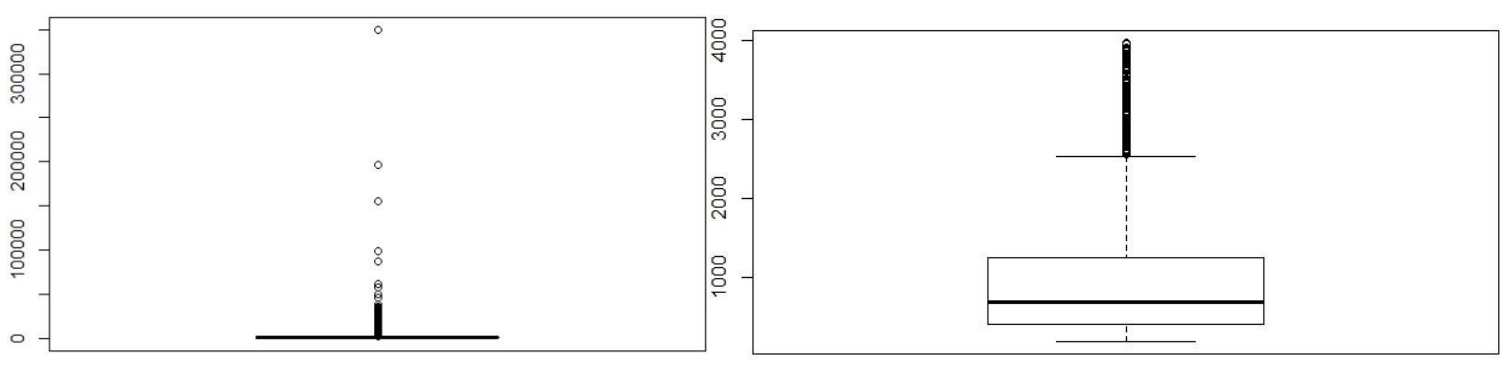

Figure 1. Price boxplot left (a) with outliers and right (b) without outliers 


\subsection{Data Exploration}

This section will seek to answer the first five research questions through the use of an exploratory data analysis performed on Mexico city's AirBnb listing dataset obtained in November of 2019.

\subsubsection{What are the Most Common AirBnb Property Types in Mexico City Available for Renting?}

An interesting attribute is the number of room types in Mexico City. To answer the question of what are the most common AirBnb property types available for rent, we plotted the number of accommodations (Figure 2.a) The frequency of room types varies between 349 and 9,238. Also, we can observe that the amount of shared rooms and hotel rooms is rare and the entire home apartments dominate the listing. Additionally, (Figure 2.b) shows that besides hotel rooms, the room types are affected by extreme values in the listings. Private rooms and shared rooms are both below the mean, while entire-home apartments and hotel rooms are around the mean or higher.

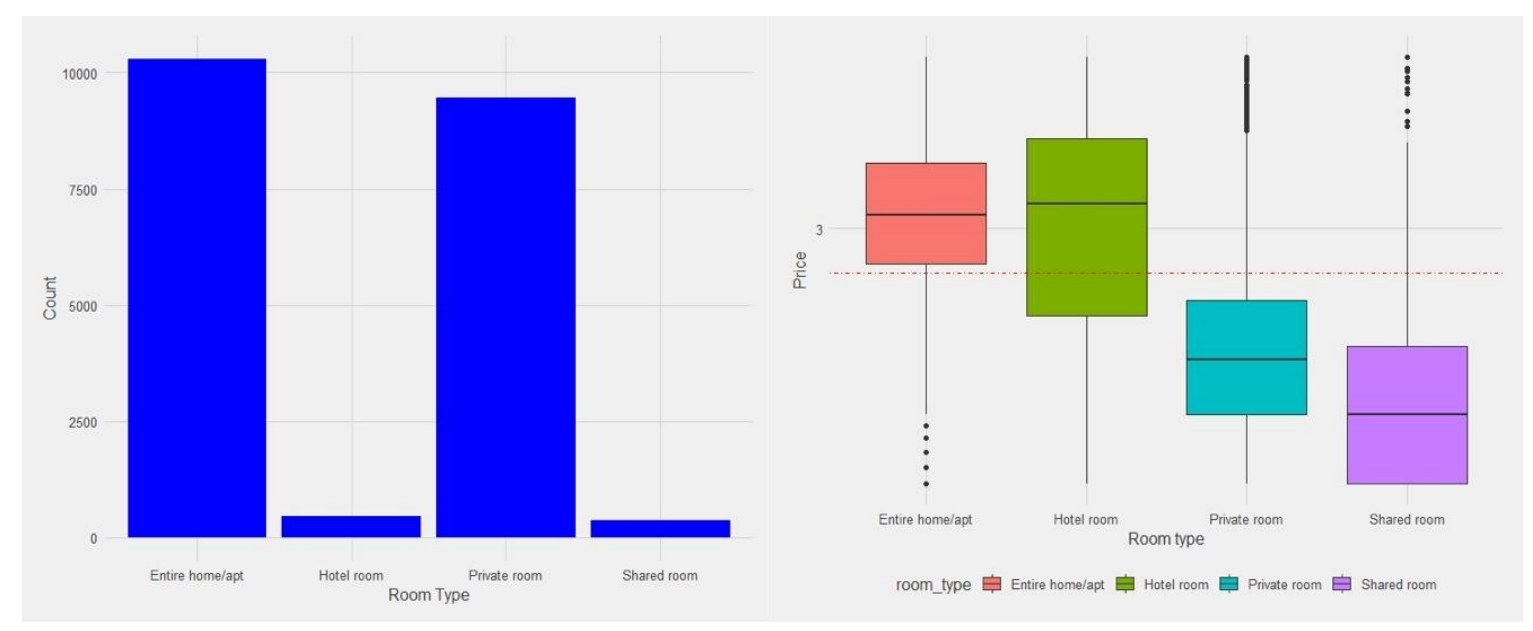

Figure 2. (a) Count of room types in left and (b) price by type room in right

\subsubsection{What is the Average Price of the Properties?}

To answer the question of what is the average price of the properties, we plotted the price distribution (Figure 3) The average price of a property in Mexico City is MXN \$991.80. Due to the skewness observed in the plot, and the violation that the data reflects when performing residual analysis of normality, we can conclude that the distribution is not normal, therefore we perform a logarithmic transformation to transform the dependent variable "Price" into a more symmetric distribution and reduce the negative effect of the skewness when performing the regression models. 


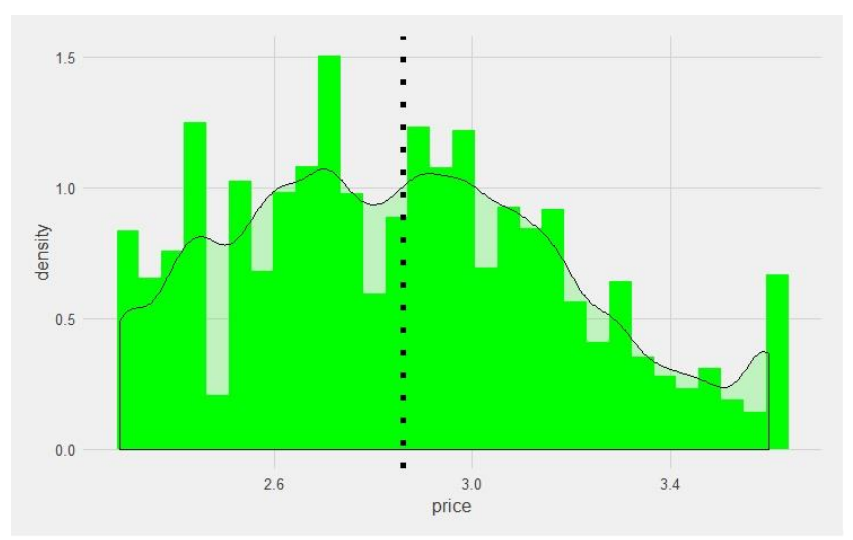

Figure 3. Price distribution logarithm scale

\subsubsection{What is the Most Expensive Location in Mexico City?}

To visualize the most expensive locations in Mexico City, we created a plot showing the density of the price per neighborhood attribute. From the plot (Figure 4) we concluded that the neighborhood "Miguel Hidalgo" has the highest AirBnb average price in the city.

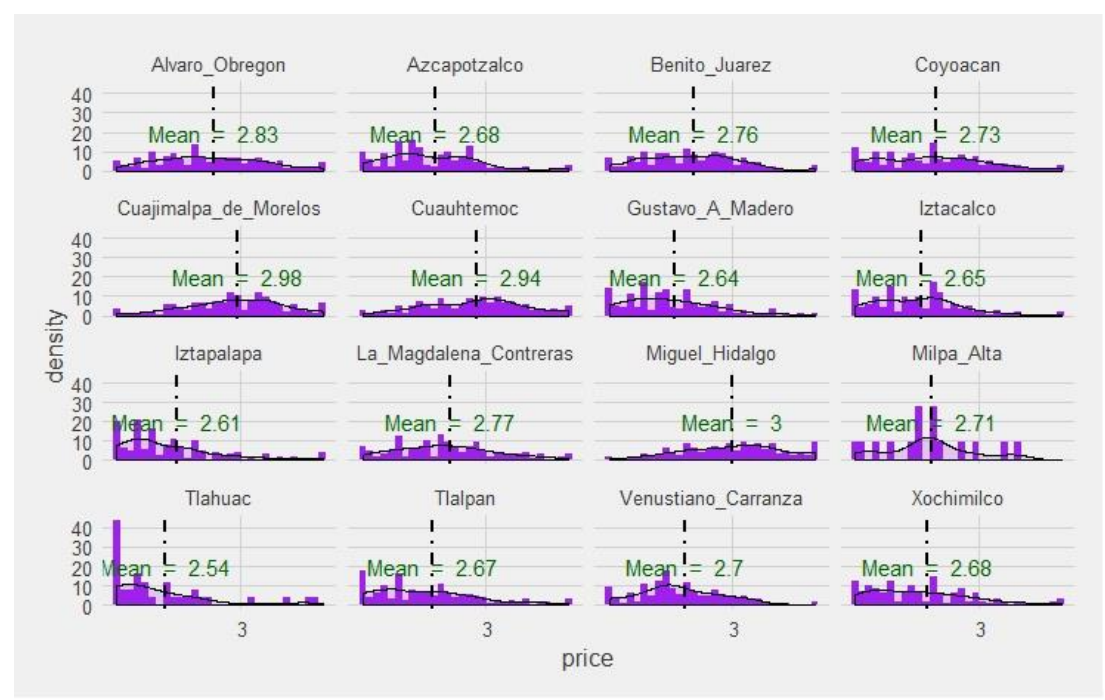

Figure 4. Price distribution by neighborhood $\log 10$

\subsubsection{What Time of the Year are AirBnb's Listings Most Popular in Mexico City? Is there any Holiday that Spikes Pricing?}

When exploring the calendar data, an interesting trend is a price in comparison to the days of the week. (Figure 5.a) shows a peak on Friday and remains stable until a fall on Sunday. This reflects how the weekends suffer from higher prices in the AirBnb listings of Mexico City. Calendar data are not used to train the predictive model, the prices of the listings are taken from November AirBnb listing prices of 2019. 


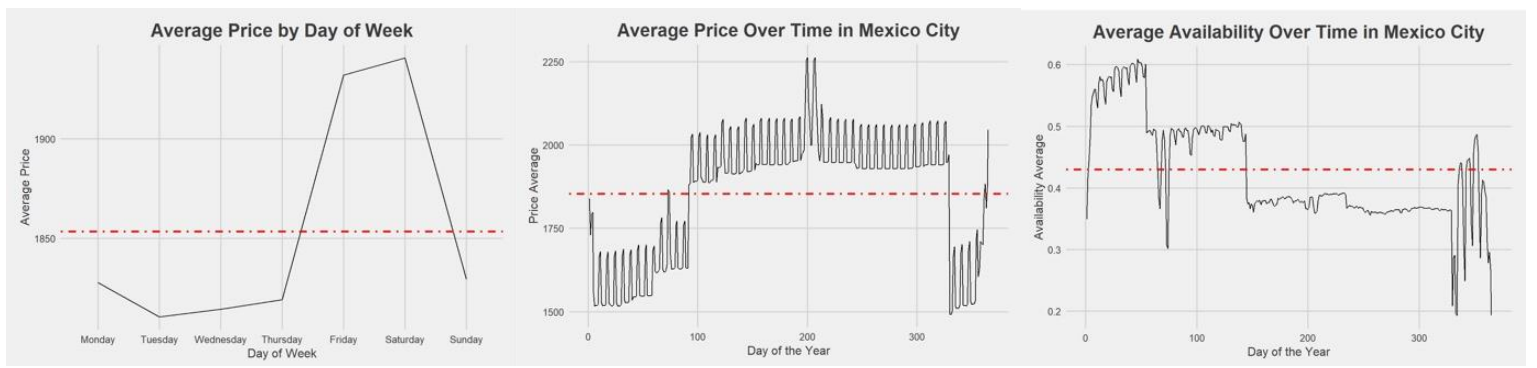

Figure 5. (a) Average price by day of the week in left, (b) average price over time in Mexico City in middle, and (c) Average availability over time in Mexico City in right

\subsubsection{What are the Most Important Features of a Listing in Mexico City, and how does this Affect the Price?}

To inspect the correlation between all variables, we built a Spearman correlation matrix (Figure 6). From the results of this matrix, we can conclude that the feature "accommodates" is strongly correlated with the price (\$> 0.5 Spearman correlation), and there are other features which are slightly positively correlated with the following features of the listing, these are: "bedrooms" (0.47), "beds" (0.44), "guests_included" $(0.33)$, "bathrooms" (0.35), and "latitude" (0.21); on the other hand "longitude" $(-0.23)$ is slightly negatively correlated. The "latitude" and "longitude" attributes indicate the coordinates where the listings are situated in Mexico City. As the latitude is positively correlated and the longitude is the other way, those listings located in the northwestern part are more expensive than those in the southeastern part of Mexico City.

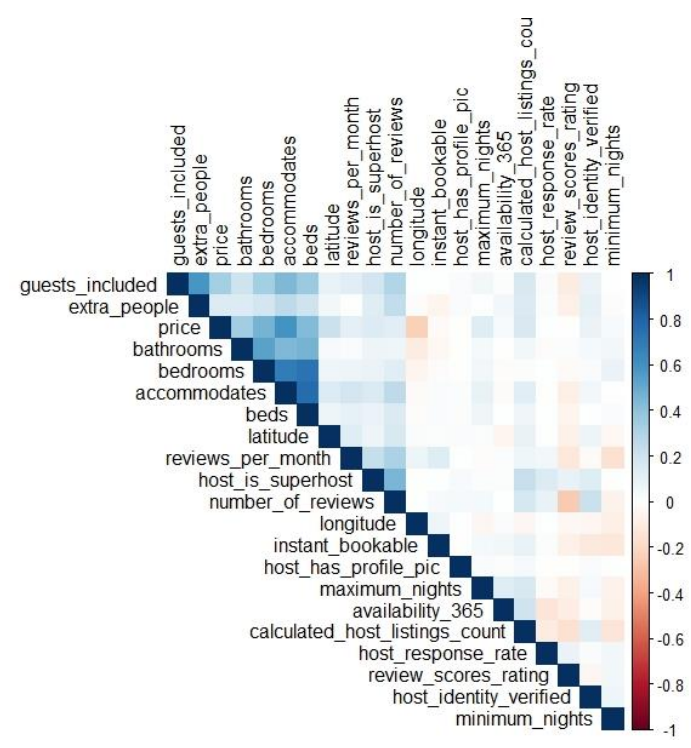

Figure 6. Spearman correlation matrix plot

\subsection{Data Transformation}

The dependent variable was transformed to a logarithmic scale to deal with skewed data. Another transformation done was feature scaling. We can observe that the data contains features with varying magnitudes, when this occurs the distance measure can overweight the features with larger values than the others. To solve this issue, we scale the data to a specific range. In this study, we use the Min-max normalization technique to obtain a distribution of values between $[0,1]$. 


\section{DATA MODELING}

This phase is the fourth of the CRISP-DM process and consists of the selection of the appropriate modeling technique.

\subsection{Quantile Regression Model}

To calculate the results of the quantile regression model, each regression model quantile is accounted for independently from each other by its percentage. In this study, we obtained a model for each of the following quantiles: $0.10,0.25,0.50,0.75$, and 0.90 . The complete list of coefficients for each quantile is not presented due to its length and the requirement of this paper's limit, however, the first 3 most representative coefficients for each quantile are shown in Table 3, the rest are available upon request.

Table 3. Quantile regression coefficients summary

\begin{tabular}{|c|c|c|c|c|c|c|}
\hline & & minimum_nights & accommodations & bedrooms & bathroom & (Intercept) \\
\hline \multirow{3}{*}{ Quantile .10 } & Estimate & -6.0845 & 1.7513 & 1.4722 & 1.1932 & 0.2343 \\
\hline & Std. Error & 0.7454 & 0.0941 & 0.1771 & 0.1807 & 0.7533 \\
\hline & P-value & 0.0000 & 0.0000 & 0.0000 & 0.0000 & 0.0019 \\
\hline \multirow{3}{*}{ Quantile .25 } & Estimate & -9.4427 & 1.9119 & 1.4041 & 1.5446 & 0.2223 \\
\hline & Std. Error & 0.7251 & 0.0628 & 0.1594 & 0.1371 & 0.0296 \\
\hline & P-value & 0.0000 & 0.0000 & 0.0000 & 0.0000 & 0.0000 \\
\hline \multirow{3}{*}{ Quantile .50 } & Estimate & -10.5326 & 1.9574 & 1.2156 & 2.1234 & 0.3293 \\
\hline & Std. Error & 1.0884 & 0.0723 & 0.1544 & 0.1475 & 0.0646 \\
\hline & $\mathrm{P}$-value & 0.0000 & 0.0000 & 0.0000 & 0.0000 & 0.0000 \\
\hline \multirow{3}{*}{ Quantile .75 } & Estimate & -12.9230 & 1.8742 & 1.0397 & 2.8419 & 0.6096 \\
\hline & Std. Error & 1.2729 & 0.0817 & 0.1967 & 0.1557 & 0.6795 \\
\hline & P-value & 0.0000 & 0.0000 & 0.0000 & 0.0000 & 0.3696 \\
\hline \multirow{3}{*}{ Quantile .90 } & Estimate & -13.4145 & 1.6431 & 1.1637 & 2.5852 & 0.9595 \\
\hline & Std. Error & 1.9573 & 0.1412 & 0.3135 & 0.2695 & 0.1242 \\
\hline & P-value & 0.0000 & 0.0000 & 0.0002 & 0.0000 & 0.0000 \\
\hline
\end{tabular}

The quantile regression graphs in Figure 7 provide a visualization of the difference in coefficients across the quantiles with their confidence intervals.
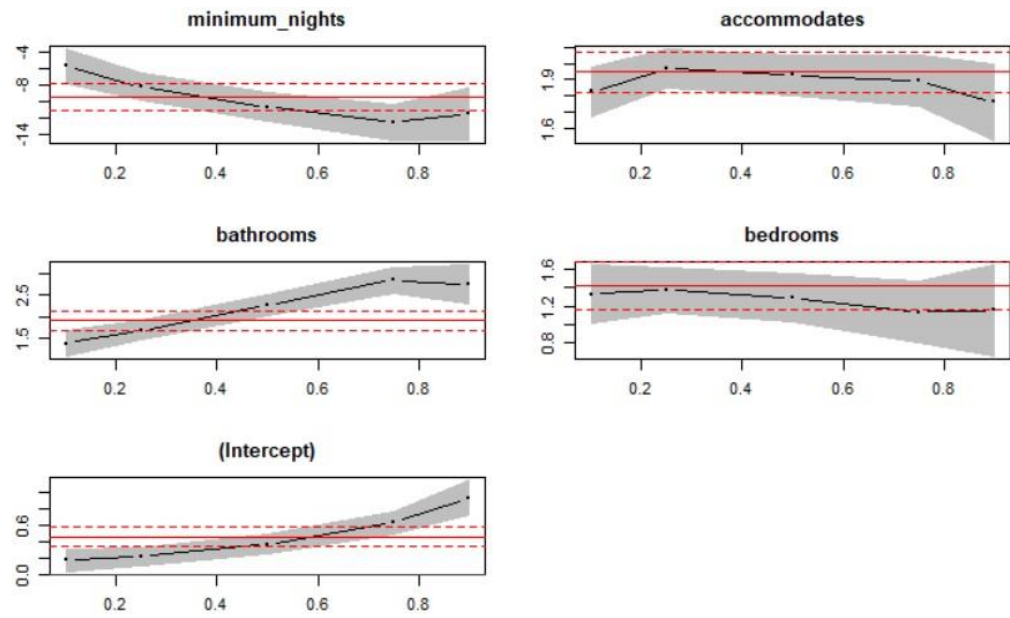

Figure 7. Quantile plot 


\subsection{Multinomial Logistic Regression Model}

Some levels can be grouped to simplify the interpretation of the model and reduce categories that contain few observations. The grouping of the attributes is as follows: the cancellation policy (3 levels) variable as flexible (10223), moderate (6013), strict (4335); host_response_time (3 levels) as within an hour (15482), within a few hours (2470), a_day_or_more(2619); the neighbourhood_cleansed (3 levels) variable as center_sector (16504), south_sector (1200), north_sector (2867); the room_type variable is left as it is and the bed_type attribute is eliminated as there are 20,411 observations with Real Bed level that represents the $99 \%$ of the bed_type category.

Before fitting the model, a one-hot encoding of the categorical variables was required. Then, the response variable column that discretizes the price of the listings was created with 3 thresholds and obtained the labels: low, medium, and the high price of listings. The low level contains prices from \$194 to \$407 MXN, the medium level contains listing prices from $\$ 426$ to $\$ 1,259$ and the high level contains listing prices from $\$ 1,278$ to $\$ 3,971$. Table 4 shows the results obtained from the multinomial logistic regression model. The model obtained an accuracy of 0.67 of correctly classified instances and an AUC of 0.84. The results of the confusion matrix are shown in Table 5.

Table 4. Logistic regression models performance summary

\begin{tabular}{cccccc}
\hline \multicolumn{5}{c}{ Logistic Regression } \\
\hline Model & AIC & Pseudo R2 & RMSE & Accuracy & AUC \\
\hdashline Binomial & 13571.00 & .49 & .36 & .82 & .89 \\
Ordinal & 24641.34 & .29 & 257.21 & .65 & .83 \\
Multinomial & 23969.99 & .31 & 1.34 & .67 & .84 \\
\hline
\end{tabular}

Table 5. Confusion matrix of the multinomial logistic regression model

\begin{tabular}{lccc}
\hline & & Predict_mlog_price \\
\hline & Low & Medium & High \\
\hline Low & 800 & 328 & 17 \\
Medium & 372 & 1402 & 205 \\
High & 40 & 418 & 531 \\
\hline
\end{tabular}

The resulting coefficients are shown in Table 6, only the three most significant are shown in this paper, but the complete model can be shared upon request.

Table 6. Quantile regression coefficients summary

\begin{tabular}{cclll}
\hline Coefficients & Dependent Variable & Estimate & Std. Error & P-value \\
\hline minimum_nights & Medium & -112.7035 & 16.48 & $7.96 E-12$ \\
& High & -178.3325 & 23.3 & $1.95 E-14$ \\
\hline accommodates & Medium & 25.8465 & 1.52 & $2 E-16$ \\
& High & 35.4447 & 1.76 & $2 E-16$ \\
\hline beds & Medium & -17.4296 & 2.03 & $<2 E-16$ \\
& High & -19.4665 & 2.33 & $2 E-16$ \\
\hline (Intercept) & Medium & -0.4706 & 0.29 & $1.06 E-01$ \\
& High & -2.7296 & 0.39 & $2.57 E-12$ \\
\hline
\end{tabular}

\subsection{Generalized Additive Model}

With the GAM, we obtained the relationship between the dependent variable (AirBnb listing price) and the independent variables. For this model, the same target labels, as in logistic regression, were used. Table 7 shows the three most important parametric coefficients of the GAM model and Table 8 shows the numerical smooth terms of the effective degrees of freedom that showed less complexity and were statistically significant for the model. Finally, Table 9 shows the evaluation results of this model. 
Table 7. GAM Parametric Coefficient Summary

\begin{tabular}{cccc}
\hline Coefficients & Estimate & Std. Error & P-value \\
\hline room_type Private Room & -0.1750 & 0.00 & $<$ E-16 \\
Room_type Shared Room & -0.2452 & 0.01 & 2 E-16 \\
Neighbourhood Center_sector & 0.0381 & 0.00 & 5.12 E-10 \\
\hline (Intercept) & 0.5096 & 0.03 & $<2$ E-16 \\
\hline & Table 8. GAM Smooth Terms Summary & \\
\hline Coefficients & edf & Ref.df & P-value \\
\hline s(maximum_nights) & 1.784 & 2.016 & 7.20 E-8 \\
\hline s(minimum_nights) & 3.760 & 4.481 & 5.95 E-15 \\
\hline s(host_response_rate) & 5.015 & 4.826 & 7.51 E-6 \\
\hline
\end{tabular}

\section{EVALUATION}

To identify the ability of the independent variables in explaining the price we use pseudo-R-square for logistic regression comparison and R2 for the regression models. In the quantile regression models, we found that the value in $R$ squared recorded the highest value in quantile 0.5 ( $R$ square $=0.55$ ), which means that the independent variables can explain up to $55 \%$ of the variation of the listing's price. The best performing model was the GAM with an R2 of 0.60 and an AIC of -13,750.50. The GAM gave the best results because it captures the nonlinear relationship between the variables. However, the multinomial logistic regression model also proved to be a good prediction model for this study, with an accuracy of 0.66 and an AUC of 0.835 . Table 9 shows the model performance summary of the constructed models.

Table 9. Models performance summary

\begin{tabular}{c|cccc}
\hline \multicolumn{5}{|c}{ Logistic Regression } \\
\hline Model & Quantile & AIC & R2 & RMSE \\
\hline & .10 & -8273.15 & .52 & .27 \\
Quantile & .25 & -11997.89 & .54 & .21 \\
Regression & .50 & -12125.63 & .55 & .18 \\
& .75 & -7370.15 & .54 & .19 \\
\hdashline GAM & .90 & 329.44 & .51 & .28 \\
\hline
\end{tabular}

\section{CONCLUSIONS}

This paper mainly concentrates on the application of machine learning models to the prediction of Mexico city’s AirBnb listing prices. It performs exploratory data analysis and compares regression models (Quantile Regression, Logistic Regression, and GAM) for price prediction. Additionally, it shows how to do a proper use of the CRISP-DM methodology for analyzing housing data and obtaining insights.

The exploratory data analysis phase of the study was able to respond to several of the research questions established in the business understanding of the Mexico AirBnb data. We obtained information regarding the highest amount of accommodation type, the most expensive neighborhood, and the average price of the properties. Additionally, with the use of visualization, we were able to get insight regarding the days of the week in which the prices rise, and the months that have lower availability and its relation with the price. Finally, we identified with the use of statistics the positive correlation between the price of a listing and its attributes.

From the results obtained from the modeling phase, the GAM obtained a better fitness score for the data when compared to the rest of the models. The most important features to predict the price obtained from this model were: 'room type', 'neighborhood', 'maximum nights', 'minimum nights', and the 'host response state'. 
Additionally, we were able to obtain better results in the quantile regression model in comparison to previous studies, which we recommend to obtain a complete picture for the observations of AirBnb listings through a number of quantile regression lines.

In comparison to the related studies, we can see that our results for the best model (GAM) obtained a lower R2 than that of Mason and Quigley (Mason and Quigley, 1996) which considered variables such as historical prices. However, since our results only consider the characteristics of the housing, we consider the results of $\mathrm{R} 2=0.6$ as a good baseline score for future analysis. Additionally, when comparing the multinomial model in the housing study from Raymond (Raymond et al., 2016), we can see that we could improve our results by integrating neighborhood characteristics such as race and poverty rates into the model. The future work for this study can include integrating these variables and exploring tree-based methods and deep learning techniques to improve the performance of the classifier. We consider this analysis as a good contribution to the existing literature about hospitality analytics introducing business methodologies and machine learning techniques that can be used in accommodation sharing platforms like AirBnb.

\section{ACKNOWLEDGEMENT}

This study was supported by the School of Engineering and Science, Tecnológico de Monterrey, Monterrey, México, and by the PNPC CONACYT program with its financial support for studying our master's degree.

\section{REFERENCES}

Aaker, D., 2019. Winning in the sharing economy: Six keys to airbnb's success, Journal of Brand Strategy, vol. 7, no. 4, pp. 310-317, 2019.

Affuso, E. et al, 2019. Is Airport Proximity an Amenity or Disamenity? An Empirical Investigation Based on House Prices. Land Economics, 95(3), 391-408.

Chapman, P. et al, 2000. CRISP-DM 1.0: Step-by-step data mining guide. SPSS inc, 9, 13.

Cox, M., 2017. Inside Airbnb: Adding data to the debate. Inside Airbnb [Internet].

Deisenroth, M. P., Faisal, A. A., \& Ong, C. S., 2020. Mathematics for machine learning. Cambridge University Press.

Dutt, S. et al, 2019. Machine Learning, 1st ed. Uttar Pradesh, India: Pearson India Education Services Pvt. Ltd.

James, G. et al, R., 2013. An introduction to statistical learning Vol. 102 New York: springer.

Jasrotia, A., \& Gangotia, A., 2018. Smart cities to smart tourism destinations: a review paper. Journal of Tourism Intelligence and Smartness, Volume 1, pp. 47-56.

Koenker, R., 2012. Quantile regression in R: A vignette. Retrieved November.

Mason, C., \& Quigley, J. M., 1996. Non-parametric hedonic housing prices. Housing studies, 11(3), 373-385.

Perez-Sanchez, V. et al, 2018. The what, where, and why of Airbnb price determinants. Sustainability, 10(12), 4596.

Raymond, E. et al, 2016. Race and uneven recovery: Neighborhood home value trajectories in Atlanta before and after the housing crisis. Housing Studies, 31(3), 324-339.

Tedjasaputra, A., \& Sari, E., 2016. Sharing economy in smart city transportation services. In Proceedings of the SEACHI 2016 on Smart Cities for Better Living with HCI and UX (pp. 32-35).

Wang, D., \& Nicolau, J. L., 2017. Price determinants of sharing economy-based accommodation rental: A study of listings from 33 cities on Airbnb. com. International Journal of Hospitality Management, 62, 120-131.

Wilcox, R., 2005. Trimming and winsorization. Encyclopedia of biostatistics, 8 .

Yu, H., \& Wu, J., 2016. Real estate price prediction with regression and classification. CS229 (Machine Learning) Final Project Reports.

Zietz, J. et al, 2008. Determinants of house prices: a quantile regression approach. The Journal of Real Estate Finance and Economics, 37(4), 317-333. 